\title{
Biochemical risk indices, including plasma homocysteine, that prospectively predict mortality in older British people: the National Diet and Nutrition Survey of People Aged 65 Years and Over
}

\author{
Christopher J. Bates ${ }^{1}$, Mohammed A. Mansoor ${ }^{2}$, Kristina D. Pentieva ${ }^{3}$, Mark Hamer ${ }^{4}$ and Gita D. Mishra ${ }^{4}$ \\ ${ }^{1}$ MRC Human Nutrition Research, Elsie Widdowson Laboratory, Fulbourn Road, Cambridge CB1 9NL, UK \\ ${ }^{2}$ Department of Natural Sciences, University of Agder, Kristiansand, Norway \\ ${ }^{3}$ Northern Ireland Centre for Food and Health, School of Biomedical Sciences, University of Ulster, Coleraine BT52 1SA, UK \\ ${ }^{4}$ Department of Epidemiology and Public Health, University College London, London WC1E 6BT, UK \\ (Received 19 November 2009 - Revised 8 March 2010 - Accepted 9 March 2010 - First published online 19 April 2010)
}

Predictive power, for total and vascular mortality, of selected indices measured at baseline in the British National Diet and Nutrition Survey (community-living subset) of People Aged 65 Years and Over was tested. Mortality status and its primary and underlying causes were recorded for 1100 (mean age 76.7 (SD 7.5) years, 50.2\% females) respondents from the baseline survey in 1994-5 until September 2008. Follow-up data analyses focussed especially on known predictors of vascular disease risk, together with intakes and status indices of selected nutrients known to affect, or to be affected by, these predictors. Total mortality was significantly predicted by hazard ratios of baseline plasma concentrations (per SD) of total homocysteine (tHcy) (95\% CI) 1.19 (1.11, 1.27), pyridoxal phosphate 0.90 (0.81, 1.00), pyridoxic acid 1.10 (1.03, 1.19), $\alpha_{1}$-antichymotrypsin $1.21(1.13,1.29)$, fibrinogen $1.14(1.05,1.23)$, creatinine $1.20(1.10,1.31)$ and glycosylated $\mathrm{Hb} 1.23$ $(1.14,1.32)$, and by dietary intakes of energy $0.87(0.80,0.96)$ and protein $0.86(0.77,0.97)$. Prediction patterns and significance were similar for primary-cause vascular mortality. The traditional risk predictors plasma total and HDL cholesterol were not significant mortality predictors in this age group, nor were the known tHcy-regulating nutrients, folate and vitamin $\mathrm{B}_{12}$ (intakes and status indices). Model adjustment for known risk predictors resulted in the loss of significance for some of the afore-mentioned indices; however, tHcy 1.34 (1.04, 1.73) remained a significant predictor for vascular mortality. Thus, total and primary vascular mortality is predicted by energy and protein intakes, and by biochemical indices including tHcy, independent of serum folate or vitamin $\mathrm{B}_{12}$.

British National Survey of older adults: Mortality prediction: Intakes and biochemical indices: Plasma homocysteine: B-vitamins and inflammatory indices

Relationships between biochemical status indices and later morbidity and mortality experience can help to predict causal relationships, and thereby to clarify physiological and pathological mechanisms that may be related to important disease risk factors in human subjects. Some indices and risk factors can be modified through lifestyle adjustment, and are thus amenable to public health intervention. Here, we have focussed on mortality outcomes of the community-living participants of the countrywide British National Diet and Nutrition Survey of People Aged 65 Years and Over, for which the fieldwork was performed in $1994-5^{(1)}$. Subsequent mortality outcomes were available from the National Health Service register of deaths up to September 2008. Because the predictive value of conventional risk factors appears to diminish with advancing age $^{(2)}$, recently, attention has been focused on the discriminative ability of novel risk markers in elderly cohorts ${ }^{(3)}$. The purpose of the present paper is to explore the predictive significance of a subset of the novel biochemical indices, measured as part of the original population surveillance protocol, with specific focus on plasma total homocysteine (tHcy), which has been associated with vascular disease incidence and mortality, and certain other disease risks ${ }^{(3-23)}$, and the functionally related nutrients folate, riboflavin, vitamin $\mathrm{B}_{12}$ and vitamin $\mathrm{B}_{6}$, together with biochemical indicators of inflammatory status, renal function and blood glucose exposure.

\section{Subjects and methods}

The survey procedures have been described in detail elsewhere $^{(1)}$; therefore, only a brief summary is given here. Originally, in 1994-5, two separate population samples were randomly selected: one from community-living people aged 65 years and over and the other from long-stay institutions. Only the community-living sample is included herein. Participants were drawn from eighty randomly selected postcode sectors in mainland Britain, allocated to four sequential 3-month fieldwork 'waves' corresponding to the four seasons, beginning in October 1994. Demographic, socio-economic

Abbreviations: HbA1c, glycosylated Hb; ICD, International Classification of Diseases; tHcy, total homocysteine.

* Corresponding author: Dr Christopher J. Bates, email chris.bates@mrc-hnr.cam.ac.uk 
and other information were obtained by a trained interviewer at the participants' home. A 4-d weighed dietary record was also obtained by the interviewer, and anthropometric indices, blood pressure and pulse rate, and after separate consent, a fasting early-morning venous blood sample were taken by a trained nurse. The blood sample was subdivided and used for a wide range of analyses. Of these, the assays ${ }^{(1,24,25)}$ that are relevant to the present study are (a) the reverse-phase high-performance liquid chromatographic assay used for measuring plasma tHcy, in which the samples were pre-treated with sodium borohydride, sulphosalicylic acid, dithioerythritol and monobromobimane ${ }^{(24)}$; (b) the microbiological assay used for measuring serum folate, which was based on the growth of chloramphenicol-resistant Lactobacillus casei and developed in-house by the Haematology Laboratory of Addenbrookes Hospital, Cambridge, UK, in 1994-5; (c) the commercial competitive protein-binding radioassay (Bio-Rad (Hercules, CA, USA) Quantaphase II Folate Radioassay kit, based on a milk protein folate binder bound to microbeads) used for measuring red cell folate; (d) the intrinsic factor-based competitive binding radioassay used for measuring vitamin $B_{12}$, which used purified gastric intrinsic factor as the selective binder, and was developed in-house by the Haematology Laboratory of Addenbrookes Hospital, Cambridge, UK, in 1994-5; (e) the chromatographic assay with fluorescence detection for measuring vitamin $\mathrm{B}_{6}{ }^{(25)}$; (f) the assay used for the determination of the activation coefficient of erythrocyte glutathione reductase, index of riboflavin status, which is the ratio of the enzyme activity with added FAD cofactor to that without added cofactor in vitro, which was developed at the MRC Dunn Nutrition Unit, Cambridge, UK, in 1994-5; (g) the specific nephelometric immunoassays used for measuring plasma $\alpha_{1}$-antichymotrypsin and fibrinogen, with the $\alpha_{1}$ antichymotrypsin assay being based on its binding to a specific commercial (Dako, Glostrup, Denmark) antibody to $\alpha_{1}$-antichymotrypsin raised in rabbits; (h) the commercial colorimetric assays used for the determination of the indices of lipid status (plasma total and HDL cholesterol and fasting TAG; Roche (Basel, Switzerland) Unimate kits); (i) the HPLC (Department of Clinical Chemistry, Addenbrookes Hospital, in-house method) for the determination of blood glucose exposure (glycosylated $\mathrm{Hb}(\mathrm{HbA} 1 \mathrm{c}))$. Participation in the available UK external quality assessment schemes for lipid, folate and vitamin $\mathrm{B}_{12}$ assays was undertaken; however, analogous quality assessment schemes were not available for most of the other indices; therefore, in-house quality assessments and inter-laboratory exchanges were undertaken in order to monitor the accuracy and stability of the assays. Where available (i.e. for plasma tHcy, cysteine, cysteinylglycine, pyridoxal phosphate, pyridoxic acid, $\alpha_{1}$-antichymotrypsin, TAG, total and HDL cholesterol, creatinine, serum folate and vitamin $\mathrm{B}_{12}$, red cell folate and erythrocyte glutathione reductase activation coefficient), all the between-run quality control sample $\mathrm{CV}$ were $\leq 11 \%$, and the mean quality control CV was $5.7 \%$.

The study was conducted according to the guidelines laid down in the Declaration of Helsinki, and all procedures involving the human subjects were approved by each of the Local Research Ethics Committees representing each of the eighty postcode sectors used. The protocol was also approved by the Ethical Committee of the MRC Dunn Nutrition Unit (of which the Micronutrient Status Laboratory is now part of
MRC Human Nutrition Research) in Cambridge. Written informed consent was obtained from all the subjects.

The present study included 1100 participants, comprising 547 men and 553 women with partial or complete data available for the analyses of interest here, all of whom agreed to be flagged on the National Register of Births and Deaths, and whose status (i.e. as still alive or registered as having died) was known in September 2008. No exclusions, other than those resulting from willingness to participate or the availability of blood samples, were imposed, and there was no evidence for sampling bias.

\section{Statistical analysis}

Cox proportional hazards models were used, with years as the time scale, to estimate the risk of mortality (all-cause and vascular disease) according to each biochemical and nutritional index. The data were censored to September 2008 in participants who survived, and were age adjusted, thereby including both the participants' post-survey duration of survival (up to September 2008) and their age at baseline, in the mortality risk calculations. Vascular disease mortality was defined according to the International Classification of Diseases (ICD) - version 9 (ICD-9) and version 10 (ICD-10), (ICD-9: 390-459; ICD-10: I01-I99), and it was limited to the primary cause of death, i.e. by excluding those with only secondary or underlying vascular causes.

In the basic multivariate model, we adjusted for potential confounders including age and sex in all the models, and then by adding other known (BMI, physical activity, alcohol consumption, receipt of welfare benefit and cigarette smoking) or observed correlates of mortality risk or disease and the nutrients that are known to influence tHcy. We adjusted for each risk factor separately in a sequential fashion.

The proportional hazards assumption was evaluated by testing the null hypothesis that the proportional hazards assumption holds for all variables simultaneously (the global test) and for each individual covariate. These were obtained from the statistical package STATA 10.0 command stphtest. The predictive power of tHcy for all-cause and vascular mortality was also tested by calculating the areas under the receiver operating characteristic curves. The areas were compared with the neutral value of 0.5 indicating risk prediction by chance alone, and $95 \%$ CI were calculated using all-cause mortality and vascular mortality $v$. survival. All the tests of statistical significance were based on two-sided probability.

\section{Results}

Of the (free-living) survey participants who gave consent for follow-up flagging of the National Health Service register of deaths, and who had provided at least one index value reported in this follow-up study, $94.5 \%$ could be accounted for in terms of known deaths plus known survivors, and it is their data that are included in this report.

Table 1 provides mean and median baseline values, subdivided by sex, for the indices explored in this report. The original survey report ${ }^{(1)}$ provided baseline index values for all the original survey participants, together with further details about the aims of the selection procedures and the methodologies used. 
Table 1. Summary of selected status indices and nutrient intakes in those survey respondents who are included in the present study (Mean values and standard deviations; medians and ranges, $n$ 1100)

\begin{tabular}{|c|c|c|c|c|c|c|c|c|c|c|}
\hline & \multicolumn{5}{|c|}{ Male } & \multicolumn{5}{|c|}{ Female } \\
\hline & $n^{*}$ & Mean & SD & Median & Range & $n^{\star}$ & Mean & SD & Median & Range \\
\hline Age (years) & & $75 \cdot 9$ & & 75 & $65-96$ & 543 & $77 \cdot 6$ & & 77 & $65-99$ \\
\hline Body weight (kg) & 551 & 75 & 12 & 75 & $39-121$ & 536 & 64 & 13 & 63 & $33-113$ \\
\hline BMI $\left(\mathrm{kg} / \mathrm{m}^{2}\right)$ & 546 & $26 \cdot 3$ & 3.7 & $26 \cdot 1$ & $16-43$ & 529 & $26 \cdot 6$ & 4.8 & $26 \cdot 2$ & $14-45$ \\
\hline Waist $(\mathrm{cm})$ & 550 & $97 \cdot 7$ & $10 \cdot 9$ & $98 \cdot 1$ & $47 \cdot 5-129$ & 537 & $87 \cdot 8$ & $11 \cdot 6$ & 86.5 & $27-131$ \\
\hline \multicolumn{11}{|l|}{ Blood pressure $(\mathrm{mmHg})$} \\
\hline Systolic & 536 & 150 & 22 & 149 & $92-222$ & 518 & 156 & 24 & 153 & $94-224$ \\
\hline Diastolic & 536 & 80 & 13 & 80 & $47-123$ & 518 & 78 & 15 & 77 & $40-137$ \\
\hline Pulse rate (per min) & 536 & 68 & 13 & 66 & $41-134$ & 518 & 72 & 11 & 71 & $46-110$ \\
\hline \multicolumn{11}{|l|}{ Biochemical indices } \\
\hline Plasma tHcy $(\mu \mathrm{mol} / \mathrm{l})$ & 361 & $16 \cdot 3$ & 7.5 & $15 \cdot 2$ & $5 \cdot 8-95 \cdot 6$ & 355 & $15 \cdot 2$ & 6.4 & $13 \cdot 8$ & $4.4-54.9$ \\
\hline Plasma Cys $(\mu \mathrm{mol} / \mathrm{l})$ & 359 & 262 & 30 & 259 & $167-393$ & 353 & 262 & 30 & 261 & $129-366$ \\
\hline Plasma cysteinylglycine $(\mu \mathrm{mol} / \mathrm{l})$ & 359 & $36 \cdot 6$ & 8.5 & $35 \cdot 3$ & $17 \cdot 2-64 \cdot 1$ & 352 & $36 \cdot 0$ & 8.6 & 34.7 & $14-66$ \\
\hline Serum folate $(\mathrm{nmol} / \mathrm{l})$ & 461 & $15 \cdot 4$ & $10 \cdot 3$ & $12 \cdot 5$ & $0.9-40.8$ & 442 & $16 \cdot 6$ & $10 \cdot 6$ & 13.4 & $2-40 \cdot 8$ \\
\hline Red cell folate $(\mathrm{nmol} / \mathrm{l})$ & 460 & 496 & 292 & 424 & $60-2216$ & 426 & 507 & 297 & 425 & $78-2357$ \\
\hline Serum vitamin $B_{12}(\mathrm{pmol} / \mathrm{l})$ & 457 & 226 & 103 & 207 & $49-737$ & 438 & 238 & 124 & 214 & $48-737$ \\
\hline Plasma pyridoxal phosphate $(\mathrm{nmol} / \mathrm{l})$ & 456 & $40 \cdot 2$ & $26 \cdot 8$ & $36 \cdot 8$ & $5-319$ & 431 & $43 \cdot 7$ & 41.7 & 34 & $6.5-397.5$ \\
\hline Plasma pyridoxic acid $(\mathrm{nmol} / \mathrm{l})$ & 457 & $17 \cdot 7$ & $10 \cdot 6$ & $15 \cdot 3$ & $4-103$ & 431 & $19 \cdot 6$ & $25 \cdot 0$ & $14 \cdot 8$ & $4.5-372.5$ \\
\hline EGRAC (ratio) & 448 & 1.31 & 0.18 & $1 \cdot 27$ & $1 \cdot 0-2 \cdot 2$ & 427 & 1.30 & $0 \cdot 16$ & 1.27 & $1.02-2.41$ \\
\hline Plasma $\alpha_{1}$-antichymotrypsin (g/l) & 445 & 0.38 & 0.10 & 0.37 & $0.16-1.15$ & 421 & 0.40 & 0.09 & 0.39 & $0.23-1.01$ \\
\hline Plasma fibrinogen $(\mathrm{g} / \mathrm{l})$ & 428 & $5 \cdot 8$ & 1.8 & 5.5 & $1 \cdot 3-11$ & 401 & $5 \cdot 8$ & 1.5 & $5 \cdot 6$ & $2-11$ \\
\hline Plasma TAG (mmol/l) & 443 & 1.57 & 0.93 & 1.33 & $0.43-7.06$ & 414 & 1.62 & 0.77 & 1.45 & $0.29-5.93$ \\
\hline Plasma total cholesterol $(\mathrm{mmol} / \mathrm{l})$ & 443 & 5.45 & 1.2 & 5.5 & $2 \cdot 1-9 \cdot 4$ & 414 & $6 \cdot 18$ & 1.5 & $6 \cdot 1$ & $2.4-11.5$ \\
\hline Plasma HDL cholesterol (mmol/l) & 443 & $1 \cdot 17$ & 0.45 & $1 \cdot 1$ & $0.49-4.09$ & 414 & 1.39 & 0.45 & 1.3 & $0.5-2.95$ \\
\hline Blood HbA1c (\%) & 451 & $5 \cdot 3$ & $1 \cdot 2$ & $5 \cdot 0$ & $3-12 \cdot 4$ & 425 & $5 \cdot 10$ & 0.93 & $5 \cdot 0$ & $3.3-11.9$ \\
\hline \multicolumn{11}{|l|}{ Estimated average daily dietary intakes } \\
\hline Energy (kJ) & 540 & 7935 & 1942 & 7949 & $3437-17301$ & 521 & 5924 & 1414 & 5864 & $1908-9769$ \\
\hline Fat $(\mathrm{g})$ & 540 & 74.0 & 23 & 72 & $20-221$ & 521 & 57 & 18 & 56 & $14-221$ \\
\hline Protein (g) & 540 & $70 \cdot 3$ & 17 & 70 & $17-124$ & 521 & $55 \cdot 0$ & 14 & 55 & $15-105$ \\
\hline Folate $(\mu \mathrm{g})$ & 540 & 263.4 & 95 & 253 & $75-728$ & 521 & 204 & 75 & 193 & $27-535$ \\
\hline Vitamin $B_{12}(\mu \mathrm{g})$ & 540 & 5.9 & 6.4 & 4.3 & $0.55-87.2$ & 521 & 4.3 & 4.4 & $3 \cdot 2$ & $0 \cdot 66-42 \cdot 8$ \\
\hline Vitamin $B_{6}(\mathrm{mg})$ & 540 & $2 \cdot 1$ & 0.7 & $2 \cdot 0$ & $0.4-6.0$ & 521 & 1.6 & 0.5 & 1.5 & $0.14-3.93$ \\
\hline Vitamin $B_{2}$ (riboflavin; mg) & 540 & 1.7 & 0.7 & 1.7 & $0.2-7.29$ & 521 & 1.4 & 0.6 & 1.3 & $0.3-3.9$ \\
\hline
\end{tabular}

tHcy, Total (plasma) homocysteine; EGRAC, erythrocyte glutathione reductase activation coefficient (index of riboflavin status; ratio of two enzyme activities); HbA1c, glycosylated $\mathrm{Hb}$ (expressed as the percentage of total $\mathrm{Hb}$ ).

"Maximum values for $n$ in Tables 1 and 2 are limited to the numbers dinis

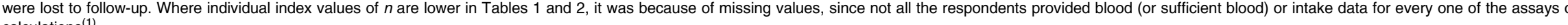


Table 2 shows the age-and sex-adjusted hazard ratios for all-cause and primary vascular disease-cause mortality for the indices of interest here. Significant predictors of all-cause mortality were plasma total tHcy, pyridoxal phosphate, $\alpha_{1}$-antichymotrypsin, fibrinogen and creatinine; blood HbA1c and dietary intakes of energy and protein. For primary vascular disease mortality, comprising about $27 \%$ of all mortality, the significant predictors were similar, with only plasma pyridoxal phosphate and energy intake failing to achieve significance at the $5 \%$ probability level. Importantly, the traditional lipid biochemical risk factors plasma total and HDL cholesterol were not significant mortality predictors either for all-cause or for primary vascular mortality.

In order to test whether all-cause mortality prediction was heavily dependent on pre-existing disease (not shown), the all-cause mortality hazard ratio analyses were repeated after elimination of each of the three subgroups of respondents with evidence of pre-existing disease: model a, those who reported 'very poor' self-assessed health at baseline (159 of 1200 eliminated); model $b$, those who survived less than 2 years after baseline (263 eliminated); and model c, those regularly taking any prescribed medication for vascular disease (617 eliminated). The only substantive change in prediction significance compared with the results shown in Table 2 was that pyridoxic acid lost significance in model $\mathrm{c}$. We concluded from this evidence that all-cause mortality prediction probably was not heavily influenced by pre-existing disease.

Elimination of the ninety-nine subjects who were taking either folic acid or vitamin $\mathrm{B}_{12}$ or vitamin $\mathrm{B}_{6}$ (or a combination of these) as dietary supplements made essentially no difference to any of the index predictions of mortality, except for pyridoxic acid, which lost significance as a result of this elimination.

Model adjustment for a number of known risk factors for all-cause mortality (not shown) did not, in most instances, seriously affect the prediction power of the indices studied. Thus, model 1 was adjusted for age, sex, BMI, physical activity, alcohol consumption, receipt of welfare benefit, cigarette smoking, energy intake, folate, vitamin $\mathrm{B}_{12}$, vitamin $\mathrm{B}_{6}$ intakes and plasma creatinine; model 2 was adjusted for age, sex, BMI, physical activity, alcohol consumption, receipt of welfare benefit, cigarette smoking, energy intake, folate, vitamin $B_{12}$, vitamin $\mathrm{B}_{6}$ intakes, plasma creatinine, plasma pyridoxal phosphate, plasma pyridoxic acid, plasma $\alpha_{1}$-antichymotrypsin, plasma fibrinogen and blood HbA1c. In model 3 but not in model 2, plasma fibrinogen lost significance, and tHcy became marginally significant, but energy intake, plasma creatinine, $\alpha_{1}$-antichymotrypsin and blood $\mathrm{HbA1c}$ all retained their significance.

Similar model adjustments were tested for primary vascular mortality. Here, plasma tHcy and energy intake remained significant predictors after the same multiple adjustments as are listed above for all-cause mortality, but plasma creatinine, fibrinogen, $\alpha_{1}$-antichymotrypsin and blood HbA1c did not.

The predictive value of tHcy alone assessed by the area under the receiver operating characteristic curve was 0.66 (95\% CI $0.43,0.88)$ for all-cause mortality, and it was 0.58 $(0 \cdot 31,0.86)$ for primary vascular mortality (receiver operating characteristic curve not shown). In the sex, age and tHcy

Table 2. Age- and sex-adjusted hazard ratios for biochemical and nutritional indices*

(Hazard ratios and $95 \%$ confidence intervals)

\begin{tabular}{|c|c|c|c|c|}
\hline & \multicolumn{2}{|l|}{$\begin{array}{l}\text { All-cause mortality died } \\
(n 749 \text {, alive } n 351)^{\star}\end{array}$} & \multicolumn{2}{|c|}{$\begin{array}{l}\text { Vascular disease mortality died } \\
\qquad(n 199 \text {, alive } n 351)^{\star}\end{array}$} \\
\hline & Age- and sex-adjusted hazard ratios & $95 \% \mathrm{Cl}$ & Age- and sex-adjusted hazard ratios & $95 \% \mathrm{Cl}$ \\
\hline \multicolumn{5}{|l|}{ Biochemical indices (per SD) } \\
\hline Plasma tHcy $(\mu \mathrm{mol} / \mathrm{l})$ & $1 \cdot 19$ & $1 \cdot 11,1 \cdot 27$ & 1.36 & $1.13,1.63$ \\
\hline Plasma Cys $(\mu \mathrm{mol} / \mathrm{l})$ & 1.06 & $0.97,1.16$ & 1.09 & $0.91,1.30$ \\
\hline Plasma cysteinylglycine $(\mu \mathrm{mol} / \mathrm{l})$ & 0.96 & $0.87,1.06$ & 0.91 & $0.75,1.11$ \\
\hline Serum folate $(\mathrm{nmol} / \mathrm{l})$ & 0.93 & $0.86,1.01$ & 0.96 & $0.82,1.12$ \\
\hline Red cell folate $(\mathrm{nmol} / \mathrm{l})$ & 0.97 & $0.90,1.05$ & 1.02 & $0.89,1.17$ \\
\hline Serum vitamin $B_{12}(\mathrm{pmol} / \mathrm{l})$ & 0.94 & $0.86,1.02$ & 0.95 & $0.80,1.12$ \\
\hline Plasma pyridoxal phosphate $(\mathrm{nmol} / \mathrm{l})$ & 0.90 & $0.81,1.00$ & 0.99 & $0.85,1.17$ \\
\hline Plasma pyridoxic acid $(\mathrm{nmol} / \mathrm{l})$ & $1 \cdot 10$ & $1.03,1.19$ & 1.17 & $1.07,1.29$ \\
\hline EGRAC (ratio) & 1.05 & $0.97,1.14$ & 0.91 & $0.77,1.08$ \\
\hline Plasma $\alpha_{1}$-antichymotrypsin $(\mathrm{g} / \mathrm{l})$ & $1 \cdot 21$ & $1.13,1.29$ & 1.21 & $1.06,1.40$ \\
\hline Plasma fibrinogen $(\mathrm{g} / \mathrm{l})$ & 1.14 & $1.05,1.23$ & $1 \cdot 16$ & $1.00,1.36$ \\
\hline Plasma TAG $(\mathrm{mm})$ & 0.96 & $0.88,1.05$ & 0.96 & $0.80,1.15$ \\
\hline Plasma total cholesterol (mmol/l) & 0.90 & $0.83,0.99$ & 0.89 & $0.73,1.08$ \\
\hline Plasma HDL cholesterol (mmol/l) & 1.02 & $0.94,1.11$ & 1.03 & $0.85,1.25$ \\
\hline Blood HbA1c (\%) & 1.23 & $1.14,1.32$ & 1.32 & $1.11,1.57$ \\
\hline Plasma creatinine & $1 \cdot 20$ & $1 \cdot 10,1 \cdot 31$ & 1.25 & $1.05,1.49$ \\
\hline \multicolumn{5}{|l|}{ Dietary data (per SD) } \\
\hline Energy (kJ) & 0.87 & $0.80,0.96$ & 0.86 & $0.72,1.02$ \\
\hline Fat $(\mathrm{g})$ & $1 \cdot 10$ & $0.94,1.29$ & 0.92 & $0.79,1.08$ \\
\hline Protein $(\mathrm{g})$ & 0.86 & $0.77,0.97$ & 0.79 & $0.67,0.94$ \\
\hline Folate $(\mu \mathrm{g})$ & 0.95 & $0.86,1.05$ & 0.88 & $0.75,1.04$ \\
\hline Vitamin $B_{12}(\mu \mathrm{g})$ & 1.01 & $0.94,1 \cdot 10$ & 0.91 & $0.74,1.11$ \\
\hline Vitamin $B_{6}(\mathrm{mg})$ & 0.91 & $0.82,1.01$ & 0.85 & $0.72,1.01$ \\
\hline Vitamin $\mathrm{B}_{2}$ (riboflavin; mg) & 0.98 & $0.90,1.06$ & 0.87 & $0.73,1.03$ \\
\hline
\end{tabular}

tHcy, Total (plasma) homocysteine; EGRAC, erythrocyte glutathione reductase activation coefficient (index of riboflavin status; ratio of two enzyme activities); HbA1c, glycosylated $\mathrm{Hb}$ (expressed as the percentage of total $\mathrm{Hb}$ ).

*As explained in the legend of Table 1, these were the study-maximum values for $n$; the actual values for each index were the same as in Table 1 . 
model for all-cause mortality, the areas under the receiver operating characteristic curve were significantly greater than those in age-sex model only $(0.73(0.69,0.77) v .0 .69(0.65$, $0.73)$ ). Similar results were obtained for vascular mortality $(0.71(0.65,0.76)$ v. $0.74(0.68,0.79)$.

The relationships between tHcy and all-cause or vascular mortality exhibited no significant deviations from linearity, suggesting that no specific tHcy threshold effects were detectable.

In view (a) of the strength of mortality prediction by both tHcy and dietary protein intake here, and (b) of a previous report $^{(26)}$ that tHcy and protein intake may be inversely correlated with each other in older adults in the USA, this inter-relationship was investigated. There was a strong inverse linear cross-sectional relationship between $\ln (\mathrm{tHcy})$ and protein intake $(t=-6 \cdot 3, P<0.0001)$ after adjustment for age and sex, which was not substantially altered by inclusion of energy intake, or by folate, vitamin $\mathrm{B}_{12}$, vitamin $\mathrm{B}_{6}$ or riboflavin in the model. There was a significant, but less strong, direct relationship between protein intake and vitamin $B_{12}$ intake, and there were similar relationships (all essentially unaffected by adjustment for energy intake) between protein intake and intakes of the other three B-vitamins.

\section{Discussion}

Important strengths of this study are that, as far as possible, the population sample was chosen as being statistically representative of the community-living people of mainland Britain in 1994-5. A wide range of nutrition-related factors were measured at baseline, including questionnaire-derived socio-demographic information, a 4-d weighed diet estimate, anthropometric measurements, haematology, blood and urine biochemistry (including a large number of nutritional indices), and dental assessment, and the follow-up period for mortality outcomes was substantial, i.e. about 14 years. One inevitable weakness, invariably associated with any cross-sectional national survey, was the fact that the baseline measures were sampled at a single time point only, although most biomarkers are thought to be sufficiently stable for use in long-term risk prediction ${ }^{(27)}$.

Our conclusion from Table 2 that plasma tHcy, whose concentration was much higher in older community-living British adults than in younger people living in the same community ${ }^{(28)}$, is also a robust and independent predictor of subsequent mortality in these older adults, both for all-cause mortality and especially for primary-cause vascular mortality, is in agreement with a considerable number of studies in Western countries during the past decade ${ }^{(3,6,8,9,12,14,17,19-23,29)}$. In agreement with certain other studies ${ }^{(3,23,30)}$, we observed no significant mortality prediction from the available blood status indices of folate or vitamin $B_{12}$ (i.e. serum folate, serum vitamin $B_{12}$ and red cell folate) or with the estimates of their intakes, even though these two vitamins are well known to be powerful modulators of plasma tHcy concentrations, including in this population sample ${ }^{(28,31)}$.

Another putative modulator of plasma tHcy concentrations, plasma pyridoxal phosphate, was a modest predictor of mortality in the present study; however, the available evidence suggests that it may reflect acute phase status more strongly than tHcy status ${ }^{(32,33)}$, and that it failed to survive into the multivariate-adjusted models described in the Results section.
The same was true for the other measured vitamins ${ }^{(32,33)}$, which also failed to survive in our highly adjusted models.

It has been claimed that tHcy may predict the risk of stroke more powerfully than it predicts that of $\operatorname{IHD}^{(34,35)}$; however, we were unable to test this particular claim in our study, because the ICD information available to us was not sufficiently cause specific. The absence of any detectable nonlinearity, or threshold effect, for tHcy with respect to its prediction of all-cause and vascular mortality in our study is consistent with the view ${ }^{(8)}$ that tHcy is a graded risk factor, whereby even a modest reduction from moderate concentrations may be beneficial.

An important question for the interpretation of the observed associations is whether the (biochemical) indices mainly reflect pre-existing disease, or acute phase status, or whether they reflect other frailty causes and their indices, such as impaired renal function. With respect to pre-existing disease, we have shown that the elimination of those respondents who were with very poor self-reported health at baseline, or of those who survived less than 2 years from baseline, or of those who were taking medication for vascular disease at baseline all made essentially no difference to the patterns and strength of prediction by the indices, including tHcy, that are described in the present paper. (It is still possible, of course, that long-term pre-existing disease, especially vascular disease, may have resulted in both raised tHcy and enhanced risk of mortality, and it is difficult, in most studies of this relationship, to determine whether observed increases in tHcy have preceded and thus truly predicted future disease risk, or whether they have been consequent upon them.) With respect to acute phase status, plasma $\alpha_{1}$-antichymotrypsin, a medium-term acute phase indicator, proved to be a robust predictor of all-cause mortality in the present study, as (to a lesser degree) did plasma fibrinogen, another index of acute phase status. A recent Dutch study ${ }^{(3)}$, in which tHcy was strongly predictive for vascular mortality in people aged $85+$ years, did not observe significant mortality predictive power for two acute phase indicators: C-reactive protein and IL-6. In contrast, Cesari et al. ${ }^{(36)}$ and two other studies quoted by them found a significant mortality prediction for C-reactive protein in older adults. In the Uppsala Longitudinal Study ${ }^{(37)}$, although none of the novel biomarkers investigated by themselves had significant impact, the addition of several biomarkers together, including C-reactive protein, substantially improved risk stratification for CVD death beyond that of conventional risk factors. Thus, the putative role for acute phase indicators in subsequent mortality prediction appears to remain uncertain. The same appears to be true for HbAlc and for other indicators of blood sugar exposure and diabeticor metabolic syndrome-status that are known to be linked to vascular risk. Our study indicated a strong and robust mortality prediction efficacy of HbA1c (Table 2); however, this was greatly attenuated if those subjects who were already, at baseline, taking drugs for the treatment of diabetes ${ }^{(38)}$ were omitted from the analysis (data not shown), and likewise, a recent study in the $\mathrm{USA}^{(39)}$ reported that $\mathrm{HbA} 1 \mathrm{c}$ failed to predict mortality in non-diabetic older adults.

In our study, three other robust predictors of all-cause mortality (Table 2) proved to be plasma creatinine, energy intake and total dietary protein intake. In older people, kidney function is a known and powerful factor with respect 
to the onset of age-related debility, and likewise, such debility is well known to lead to reductions in appetite and physical activity $^{(40)}$, which is linked in turn to reduced energy expenditure, and thus probably helps to account for the observed associations between all-cause mortality and baseline food energy and protein intakes. A prediction of acute coronary events by relatively low protein intakes was described previously $^{(41)}$. A study of retired school teachers in the USA has reported an independent inverse relationship between tHcy and protein intake ${ }^{(26)}$, and a similar relationship was observed in the present study, suggesting that dietary protein may have an important modulating effect on tHcy in older people. In our study, there was a moderate direct relationship between protein intake and intakes of vitamin $\mathrm{B}_{12}$ and of the other three B-vitamins studied here.

In conclusion, our present study has clearly demonstrated that, in agreement with other recent studies of older adults in Western societies, total plasma tHcy was a strong and independent predictor of early all-cause and vascular mortality in older adults living in the British community. Moreover, this property of tHcy appeared to be independent of its established modulatory relationships with folate and vitamin $B_{12}$ intakes, and their status indices. Other robust predictors of all-cause mortality in the present study included plasma $\alpha_{1}$-antichymotrypsin (an acute phase indicator), plasma creatinine (an index of renal status), HbA1c (an index of blood glucose exposure), and energy intake and protein intakes, which were all measured at baseline. Future intervention priorities in ageing populations will be based on an improved understanding of critical mortality risk factors, and of their response to individual- and community-based interventions.

\section{Acknowledgements}

There are no conflicts of interest. Funding was provided by the Medical Research Council. All the five authors were active in preparing the manuscript, and in addition, G. D. M. undertook the statistical analyses, and M. A. M. and K. D. P. undertook the analyses and interpretation of the tHcy and vitamin $\mathrm{B}_{6}$ analyses, respectively. The survey was commissioned jointly by the Department of Health and the Ministry of Agriculture, Fisheries and Food, whose survey responsibility has since been transferred to the Food Standards Agency. It was carried out by the National Centre for Social Research, formerly Social and Community Planning Research, in conjunction with the Micronutrient Status Laboratory of the MRC Dunn Nutrition Unit, now part of MRC Human Nutrition Research. The survey datasets were obtained from the survey commissioners, the University of Essex Data Archive and the Social Survey Division of the Office for National Statistics. We are indebted to Claire Deverill and Marie Sanchez (National Centre for Social Research) for their assistance in obtaining the mortality data.

\section{References}

1. Finch S, Doyle W, Lowe C, et al. (1998) National Diet and Nutrition Survey: People Aged 65 Years or Over. vol. 1: Report of the Diet and Nutrition Survey. London: The Stationery Office.

2. Kannel WB (2002) Coronary heart disease risk factors in the elderly. Am J Geriatr Cardiol 11, 101-107.
3. de Ruijter W, Westendorp RGJ, Assendelft WJJ, et al. (2009) Use of Framingham risk score and new biomarkers to predict cardiovascular mortality in older people: population based observational cohort study. BMJ 338, a3083.

4. Perry I, Refsum H, Morris R, et al. (1996) Serum total homocysteine and coronary heart disease in middle-aged British men. Heart 75, Suppl. 1, 53 (abstr).

5. Verhoef P, Stampfer MJ, Buring JE, et al. (1996) Homocysteine metabolism and risk of myocardial infarction: relation with vitamins $\mathrm{B}_{6}, \mathrm{~B}_{12}$, and folate. Am J Epidemiol 143, 845-859.

6. Nygard O, Nordrehaug JE, Refsum H, et al. (1997) Plasma homocysteine levels and mortality in patients with coronary artery disease. $N$ Engl J Med 337, 230-236.

7. Homocysteine-Lowering Triallists' Collaboration (1998) Lowering blood homocysteine with folic acid based supplements: meta-analysis of randomised trials. BMJ 316, 894-898.

8. Bostom AG, Silbershatz H, Rosenberg IH, et al. (1999) Nonfasting plasma total homocysteine levels and all-cause and cardiovascular disease mortality in elderly Framingham men and women. Arch Intern Med 159, 1077-1080.

9. Kark JD, Selhub J, Adler B, et al. (1999) Nonfasting plasma total homocysteine level and mortality in middle-aged and elderly men and women in Jerusalem. Ann Intern Med 131, $321-330$.

10. Whincup P, Refsum H, Perry I, et al. (1999) Serum total homocysteine and coronary heart disease: prospective study in middle aged men. Heart 82, 448-454.

11. Anderson JL, Muhlestein JB, Horne BD, et al. (2000) Plasma homocysteine predicts mortality independently of traditional risk factors and C-reactive protein in patients with angiographically defined coronary artery disease. Circulation 102, 1227-1232.

12. Omland T, Samuelsson A, Hartford M, et al. (2000) Serum homocysteine as an indicator of survival in patients with acute coronary syndromes. Arch Intern Med 160, 1834-1840.

13. Zhao X-Q, Kosinski A, Malinow MR, et al. (2000) Association of total plasma homocyst(e)ine levels and 8-years mortality following PTCA or CABG in EAST. Circulation 102 (abstr 3380).

14. Vollset SE, Refsum H, Tverdal A, et al. (2001) Plasma total homocysteine and cardiovascular and noncardiovascular mortality: the Hordaland Homocysteine Study. Am J Clin Nutr 74, 130-136.

15. Blacher J, Benetos A, Kirzin JM, et al. (2002) Relation of plasma total homocysteine to cardiovascular mortality in a French population. Am J Cardiol 90, 591-595.

16. Zylberstein DE, Bengtsson C, Bjorkelund C, et al. (2004) Serum homocysteine in relation to morbidity and mortality from coronary heart disease: a 24-year follow-up of the population study of women in Gothenburg. Circulation 109, 601-606.

17. Virtanen JK, Voutilainen S, Alfthan G, et al. (2005) Homocysteine as a risk factor for CVD mortality in men with other CVD risk factors. J Intern Med 257, 255-262.

18. Menon V, Sarnak MJ, Greene T, et al. (2006) Relationship between homocysteine and mortality in chronic kidney disease. Circulation 113, 1572-1577.

19. Refsum H, Nurk E, Smith AD, et al. (2006) The Hordaland Homocysteine Study: a community-based study of homocysteine, its determinants, and associations with disease. Am J Clin Nutr 136, 1731S-1740S.

20. Rossi GP, Maiolino G, Seccia TM, et al. (2006) Hyperhomocysteinemia predicts total and cardiovascular mortality in high-risk women. J Hypertens 24, 851-859.

21. Selhub J (2006) The many facets of hyperhomocysteinemia: studies from the Framingham cohorts. $J$ Nutr 136, 1726S-1730S

22. Gonzalez S, Huerta JM, Fernandez S, et al. (2007) Homocysteine increases the risk of mortality in elderly individuals. Br J Nutr 97, 1138-1143. 
23. Dangour AD, Breeze E, Clarke R, et al. (2008) Plasma homocysteine, but not folate or vitamin B-12, predicts mortality in older people in the United Kingdom. J Nutr 138, 1121-1128.

24. Mansoor M, Svardal A \& Ueland P (1992) Determination of the in vivo redox status of cysteine, cysteinylglycine, homocysteine and glutathione in human plasma. Anal Biochem 200, 218-229.

25. Bates CJ, Pentieva KD, Matthews N, et al. (1999) A simple, sensitive and reproducible assay for pyridoxal phosphate in human plasma. Clin Chim Acta 280, 101-111.

26. Stolzenberg-Solomon RZ, Miller ER, Maguire MG, et al. (1999) Association of dietary protein intake and coffee consumption with serum homocysteine concentrations in an older population. Am J Clin Nutr 69, 467-475.

27. Danesh J, Wheeler JG, Hirschfield GM, et al. (2004) C-reactive protein and other circulating markers of inflammation in the prediction of coronary heart disease. $N$ Engl J Med 350, 1387-1397.

28. Bates CJ, Mansoor MA, Gregory J, et al. (2002) Correlates of plasma homocysteine, cysteine and cysteinyl-glycine in respondents in the British National Diet and Nutrition Survey of young people aged 4-18 years, and a comparison with the survey of people aged 65 years and over. Br J Nutr 87, 71-79.

29. Haim M, Tanne D, Goldbourt U, et al. (2006) Serum homocysteine and long-term risk of myocardial infarction and sudden death in patients with coronary heart disease. Cardiology 107, $52-56$

30. Jia X, Aucott LS \& McNeill G (2007) Nutritional status and subsequent all-cause mortality in men and women aged 75 years or over living in the community. Br J Nutr 98, 593-599.

31. Bates CJ, Mansoor MA, van der Pols JC, et al. (1998) Plasma total homocysteine in 972 British men and women aged 65 years and over. Eur J Clin Nutr 51, 691-697.

32. Bates CJ, Pentieva KD \& Prentice A (1999) An appraisal of vitamin $\mathrm{B}_{6}$ status indices and associated confounders, in young people aged 4-18 years and in people aged 65 years and over, in two national British surveys. Public Health Nutr 2, 529-535.

33. Bates CJ, Pentieva KD, Prentice A, et al. (1999) Plasma pyridoxal phosphate and pyridoxic acid and their relationship to plasma homocysteine in a representative sample of British men and women aged 65 years and over. Br J Nutr 81, 191-201.

34. Homocysteine Studies Collaboration (2002) Homocysteine and risk of ischemic heart disease and stroke: a meta-analysis. JAMA 288, 2015-2022.

35. Wald DS, Law M \& Morris JK (2002) Homocysteine and cardiovascular disease: evidence on causality from a metaanalysis. BMJ 325, 1202-1206.

36. Cesari M, Onder G, Zamboni V, et al. (2009) C-reactive protein and lipid parameters in older persons aged 80 years and older. $J$ Nutr Health Aging 13, 587-593.

37. Zethelius B, Berglund L, Sundström J, et al. (2008) Use of multiple biomarkers to improve the prediction of death from cardiovascular causes. N Engl J Med 358, 2107-2116.

38. Bates CJ, Lean MEJ, Mansoor MA, et al. (2004) Nutrient intakes: biochemical and risk indices associated with type 2 diabetes and glycosylated haemoglobin, in the British National Diet and Nutrition Survey of people aged 65 years and over. Diabet Med 21, 677-684.

39. Chonchol M, Katz R, Fried LF, et al. (2010) Glycosylated hemoglobin and the risk of death and cardiovascular mortality in the elderly. Nutr Metab Cardiovasc Dis 20, 15-21.

40. Hamer M, Bates CJ \& Mishra GD (2010) Depressive symptoms, physical function, and risk of mortality: National Diet and Nutrition Survey in older adults $65+$ years. Am J Geriatr Psychiat (In the Press).

41. Virtanen JK, Voutilainen S, Rissanen TH, et al. (2005) High dietary methionine intake increases the risk of acute coronary events in middle-aged men. Nutr Metab Cardiovasc Dis 16, $113-120$. 